ARTICLE

Received 19 Aug 2016 | Accepted 19 Jan 2017 | Published 10 Mar $2017 \quad$ DOl: 10.1038/ncomms14650 OPEN

\title{
Engineering fungal de novo fatty acid synthesis for short chain fatty acid production
}

Jan Gajewski ${ }^{1, \star}$, Renata Pavlovic ${ }^{2, \star}$, Manuel Fischer ${ }^{1}$, Eckhard Boles ${ }^{2} \&$ Martin Grininger ${ }^{1}$

Fatty acids (FAs) are considered strategically important platform compounds that can be accessed by sustainable microbial approaches. Here we report the reprogramming of chain-length control of Saccharomyces cerevisiae fatty acid synthase (FAS). Aiming for short-chain FAs (SCFAs) producing baker's yeast, we perform a highly rational and minimally invasive protein engineering approach that leaves the molecular mechanisms of FASs unchanged. Finally, we identify five mutations that can turn baker's yeast into a SCFA producing system. Without any further pathway engineering, we achieve yields in extracellular concentrations of SCFAs, mainly hexanoic acid $\left(C_{6}-F A\right)$ and octanoic acid $\left(C_{8}-F A\right)$, of $464 \mathrm{mg} \mathrm{I}^{-1}$ in total. Furthermore, we succeed in the specific production of $\mathrm{C}_{6}$ - or $\mathrm{C}_{8}$ - $\mathrm{FA}$ in extracellular concentrations of 72 and $245 \mathrm{mgl}^{-1}$, respectively. The presented technology is applicable far beyond baker's yeast, and can be plugged into essentially all currently available FA overproducing microorganisms.

\footnotetext{
${ }^{1}$ Institute of Organic Chemistry and Chemical Biology, Buchmann Institute for Molecular Life Sciences, Cluster of Excellence 'Macromolecular Complexes', Goethe University Frankfurt, Max-von-Laue-Strasse 15, 60438 Frankfurt, Germany. ${ }^{2}$ Institute of Molecular Biosciences, Department of Biological Sciences, Goethe University Frankfurt, Max-von-Laue-Strasse 9, 60438 Frankfurt, Germany. * These authors contributed equally to this work. Correspondence and requests for materials should be addressed to E.B. (email: e.boles@bio.uni-frankfurt.de) or to M.G. (email: grininger@chemie.uni-frankfurt.de).
} 
F atty acids (FAs) are important molecules serving several purposes in living entities; that is, they are building blocks of membranes, signalling molecules and energy depots ${ }^{1,2}$. In the production of fine chemicals from renewable resources, FAs can play a crucial role as platform compounds $s^{3,4}$. Accordingly, the production of FAs in bacteria and fungi has been investigated extensively, constantly overcoming technical boundaries. In Saccharomyces cerevisiae, titres of $400 \mathrm{mgl}^{-1}$ free $\mathrm{FAs}^{3}$, and more recently of $2.2 \mathrm{gl}^{-1}$ (ref. 5) and even up to $10.4 \mathrm{gl}^{-1}$ in a glucose limited fed-batch cultivation ${ }^{6}$ have been achieved (mainly long FAs, from $\mathrm{C}_{12}$ to $\mathrm{C}_{18}$ in saturated and monounsaturated form). In E. coli, titres of up to $5.2 \mathrm{gl}^{-1}$ (predominantly $\mathrm{C}_{14}$ and $\mathrm{C}_{16: 1}$; in $\mathrm{C}_{n: m}$ the $n$ represents the number of carbons in the FA, $m$ stands for the desaturated bonds) were reported ${ }^{7}$. The native FA product spectrum of living organisms is covering mainly $\mathrm{C}_{14^{-}}$to $\mathrm{C}_{18^{-}} \mathrm{FA}$ (ref. 2), which restricts microbial production to long FAs. As FAs in the range of $\mathrm{C}_{4}$ to $\mathrm{C}_{12}$ are technologically relevant, for example as precursors for biofuels in the petrol range ${ }^{8}$, substantial efforts have been invested for harnessing bacterial and fungal fatty acid synthases (FASs) for the microbial production of short-chain fatty acids (SCFAs). Recent approaches employing thioesterases with known specificities for short-chain products have succeeded in the production of SCFAs in S. cerevisiae with titres of up to $111 \mathrm{mgl}^{-1}$ (ref. 5), and also have achieved the production of FA derived alkanes in E. coli ${ }^{4}$. Both approaches were based on interfering in native synthesis with a short acyl-ACP-specific thioesterase, which was either provided as separate protein or as a domain fused to FAS. Another more recent approach succeeded in producing $\mathrm{C}_{12^{-}}$and $\mathrm{C}_{14}$-FA with fungal FAS, and short acyl-ACP thioesterases similarly provided either as separate proteins or as domains inserted in the protein complex 9

We recently started the project of rewriting chain-length control in fungal de novo fatty acid synthesis, encouraged by the idea of performing a rational engineering approach that is minimally invasive to the protein's molecular mechanisms. The fungal FAS is a highly efficient FA producing machinery that runs synthesis at higher turnover numbers than other type I FAS systems (18.2, 2.0 and 3.4 cycles per second; values per set of active sites calculated from specific activities reported for S. cerevisiae ${ }^{10}$, Corynebacterium ammoniagenes ${ }^{11}$ and chicken $\mathrm{FAS}^{12}$, respectively). The synthetic advantage of the fungal FAS lies in its highly developed architecture, in which the catalytic domains are embedded in an extensive scaffolding matrix, and substrate shuttling by the acyl carrier protein (ACP) is subtly balanced between electrostatic steering and molecular crowding properties ${ }^{13-15}$ (Fig. 1a). In focusing on engineering strategies that address substrate specificities, but leave the overall structural properties of the fungal FAS unchanged, we aimed at steering the systems towards the premature release of the not yet completely elongated acyl-chain, while conserving the intrinsic efficiency of fungal de novo FA synthesis (Fig. 1b). In our approach, we specifically took advantage of the tight coverage of S. cerevisiae FAS with structural and functional data. The 2.6 MDa S. cerevisiae protein is the best studied fungal FAS, documented for example by a wealth of X-ray structural data at a resolution of up to $3.1 \AA$ (refs 16-21). Finally, we show that a rationally engineered $S$. cerevisiae FAS, manipulated by up to only five active site mutations, and placed in a yeast wild-type background without any metabolic pathway engineering, is extremely efficient in SCFA production. Strains carrying such engineered FASs give access to specific production of hexanoic acid $\left(\mathrm{C}_{6}-\mathrm{FA}\right.$; titres of $71.5 \mathrm{mgl}^{-1}$ corresponding to $78.2 \%$ of the detected SCFAs $\left(\mathrm{C}_{6}\right.$ to $\left.\mathrm{C}_{12}\right)$ in that strain) and of octanoic acid $\left(\mathrm{C}_{8}\right.$-FA; $\left.245.0 \mathrm{mgl}^{-1} ; 85.9 \%\right)$, and allow maximum titres of extracellular free $\mathrm{C}_{6^{-}}$to $\mathrm{C}_{12}$-FA of $464.4 \mathrm{mgl}^{-1}$. In addition,

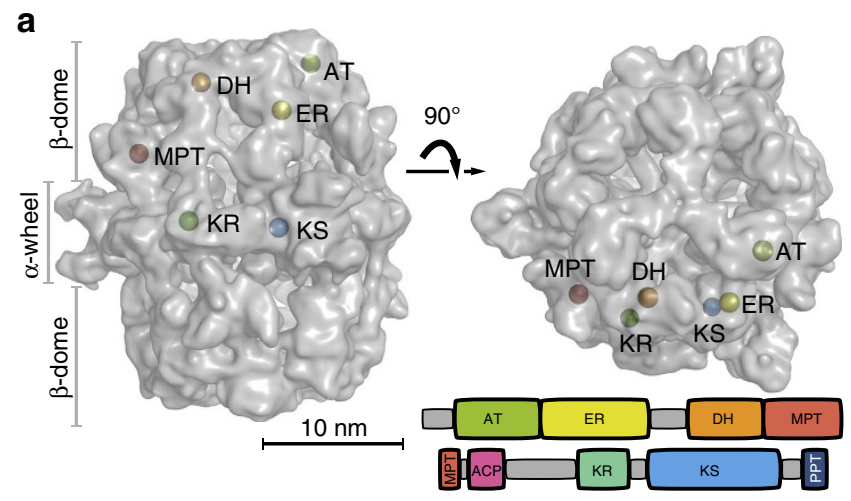

b

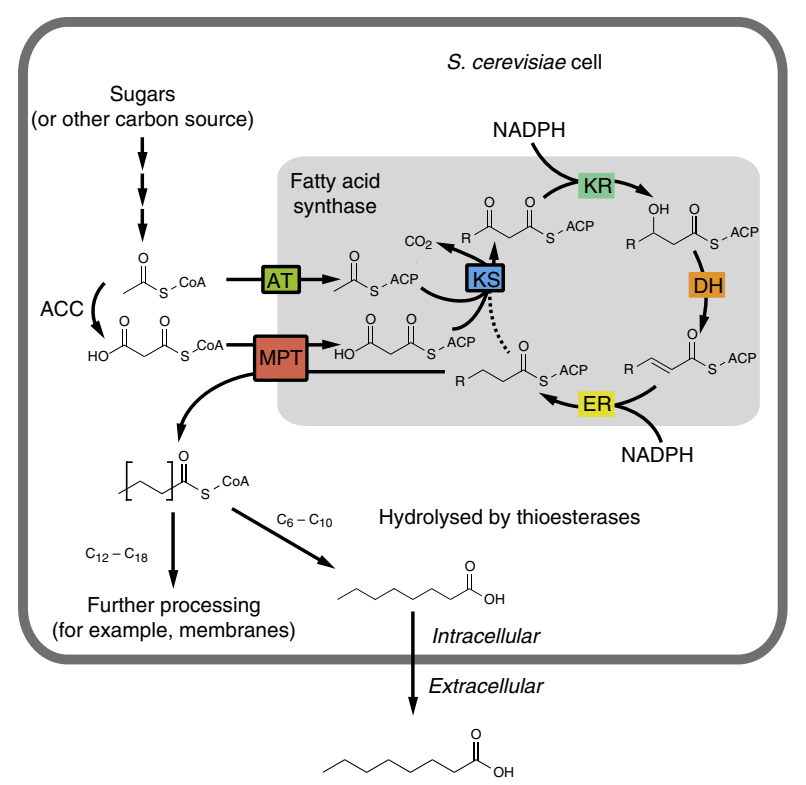

Figure 1 | Fatty acid synthesis in S. cerevisiae. (a) Two polypeptides, the $207 \mathrm{kDa} \alpha$-chain and the $229 \mathrm{kDa} \beta$-chain, assemble to a barrel-shaped $\alpha_{6} \beta_{6}$-heterododecameric complex that encloses two reaction chambers, each formed by a $\beta$-dome and the $\alpha$-wheel. The $\beta$-chain harbours the domains acetyl transferase (AT), enoyl reductase (ER), dehydratase (DH) and malonyl/palmitoyl transferase (MPT), and the $\alpha$-chain the domains acyl carrier protein (ACP), ketoacyl reductase (KR), ketoacyl synthase (KS) and the phosphopantetheine transferase (PPT). The barrelshaped structure of $S$. cerevisiae FAS is shown with one set of active centres indicated (based on protein data bank (PDB) code 3HMJ (ref. 50)). (b) In the FA cycle, the KS domain is responsible for the condensation of an acyl substrate (either the starter molecule acetyl or an ACP-bound elongated acyl-chain) with malonyl. The resulting $\beta$-ketoacyl intermediate is then processed in a series of reaction steps, performed by the domains $K R, D H$ and ER, to a fully reduced acyl-chain. The resulting acyl-chain serves as a starter for the next cycle. The process is repeated until the final product is cleaved off. Fungal FASs carry ACP domains that are double tethered to the FAS scaffold by unstructured linkers, and shuttle covalently bound substrates and intermediates to the active sites. FAS engineering aimed at producing SCFAs, instead of its native product, typically $\mathrm{C}_{16}$ - or $\mathrm{C}_{18}$-CoA. S. cerevisiae FAS naturally produces CoA esters. Free SCFAs, appearing as hydrolysed by unspecific TEs (here shown exemplarily for $\mathrm{C}_{8}$ ), are then exported to the medium, from which they have been extracted for analysis. The fatty acid cycle is shown in a schematic manner and incomplete in the presentation of side products.

this approach gives access to short-chain acyl-CoA esters, as the immediate products of fungal FA synthesis (Fig. 1b). This is highly advantageous when the desired product portfolio should 
extend towards short-chain aldehydes, alcohols and alkanes, as acyl-CoAs can then directly be processed without prior (re)activation of free $\mathrm{FAs}^{4,6,9}$. We identify three thioesterases, responsible for the hydrolysis of short-chain acyl-CoA esters in our strains. Thioesterase knockout strains hold out the prospect of efficiently channelling the short-chain acyl-CoA products into such downstream derivatization processes (Fig. 1b).

\section{Results}

Outline of approach. For manipulating chain-length regulation in the de novo production of SCFAs, key active sites of the condensation domain (KS) and the transferase domains (MPT and AT) were rationally engineered. The other domains (KR, DH and ER), which presumably have no direct influence on chain-length regulation, were not mutated (Fig. 1b). For the evaluation of FAS engineering, a $\Delta$ fas $1 \quad \Delta$ fas 2 strain was complemented with vectors carrying $\alpha$ - and $\beta$-chain encoding sequences FAS2 and FAS1, respectively. The impact of mutations was generally judged with FAS2 and FAS1 under control of their natural promoters and terminator ${ }^{22}$. SCFAs are exported and accumulate in the culture medium ${ }^{23}$, where they have been characterized after extraction with gas chromatographic methods (for more information, see Methods).

Engineering of the KS-mediated elongation step. The KS domain was considered as the most promising target for rewriting chain-length regulation, as accounting for the elongation of the growing acyl-chain (Fig. 1b). The condensation reaction is performed in a ping-pong mechanism, in which the acyl-intermediate is loaded into the KS binding channel, where it is covalently bound to the active cysteine C1305 (ping step) ${ }^{24}$. Our engineering approach was mainly informed by sequence alignments and structural data, which have been collected on KS domains ${ }^{19,25}$. We mainly focused on M1251, which is protruding into the KS binding channel and dividing the binding channel into an outer and inner volume. On acyl binding, the methionine rotates and gives access to the inner cavity (Fig. 2a). Residues at this position in S. cerevisiae FAS and in a homologous protein have been suggested to act as gate keepers that prevent short-chain acyl intermediates from KS-mediated elongation, steering them towards release ${ }^{19,25}$. To build on such an effect, we started KS engineering by introducing the mutation G1250S. S. cerevisiae strains with this mutation were characterized as producing higher levels of $\mathrm{C}_{6}$ - $\mathrm{FA}$ and its ester derivatives ${ }^{26}$, presumably by restricting the conformational flexibility of the neighbouring M1251. As a second position in the KS domain, we directly mutated M1251 with the intention to increase the steric barrier (Fig. 2a). In comparison to the wild type, the G1250S strain showed a nine-fold increase in $\mathrm{C}_{6}$-FA production $\left(15.3 \mathrm{mgl}^{-1}\right)$. The G1250S-M1251W double-mutated S. cerevisiae strain showed further increased levels of $\mathrm{C}_{6}-\mathrm{FA}$ (with $19.9 \mathrm{mgl}^{-1}, 12$-fold increase over wild type) and $\mathrm{C}_{8}$-FA (32.7 $\mathrm{mgl}^{-1}$, 56-fold increase over wild type; Fig. 2b, Supplementary Fig. 1 and Supplementary Table 1). A third promising KS mutation was identified in sequence alignments with FASs of reported $\mathrm{C}_{6}$-FA producers, such as Aspergillus nidulans, Aspergillus parasiticus and Aspergillus flavus ${ }^{27,28}$. The residue F1279 is protruding into the KS binding channel from the opposite side of G1250S-M1251W (Fig. 2a). Introducing F1279Y into a G1250S-mutated strain strongly affected growth, and FA output spectra were hardly interpretable in chain-length distribution. Beneficial effects of F1279Y were observed in combination with transferase active site mutations, as reported below (Fig. 2b).
Engineering of FAS-loading and -unloading. In fungal FAS, the processes of loading of malonyl and unloading of the fully elongated acyl-chain are performed by the MPT domain, in which all substrates are transferred via the same active serine S1808 (ref. 29) (Fig. 1b). As the MPT domain has been shown to broadly accept acyl chains of various chain lengths ${ }^{30}$, we engineered MPT for reduced malonyl affinity. As a rationale behind this mutation, a disfavoured malonyl uptake was anticipated to favour the competing release of incompletely elongated short acyl chains. Guided by mutational analysis of homologous proteins ${ }^{31,32}$, a R1834K mutation was installed to lower the affinity of malonyl by weakening the interaction with the carboxy group (Fig. 2c). The R1834K mutation led to extracellular SCFA (mostly $\mathrm{C}_{8}$ ) levels of $100 \mathrm{mgl}^{-1}$ that correspond to a 23-fold increase over wild-type FAS (Fig. 2b).

Fungal FASs catalyse loading of acetyl via the AT domain (Fig. 1b). Previous studies on related transferases reported on elevated acetyl loading after introduction of an arginine to alanine active site mutation $^{31,32}$. We expected that the corresponding I306A mutation is beneficial for SCFA synthesis (particularly in combination with decreased malonyl loading by the R1834K mutation), as elevating acetyl/malonyl ratios can lead to a pronounced priming of FA synthesis ${ }^{33,34}$. Moreover, we considered the I306A mutation as interesting, because of the reported additional effect of broadening substrate specificities of transferases towards short acyl chains ${ }^{31,32}$ (Fig. 2c). The product spectra of $S$. cerevisiae carrying the single AT mutation I306A showed no significant effect on SCFA yield. However, in combination with other mutations, I306A-mutated AT drastically changed product distributions (Fig. 2b). In Fig. 2d, putative fluxes in FA synthesis are depicted that are underlying FA production by the respective yeast strains.

Combinations of mutations. Mutations were also tested in several combinations, leading to increased amounts of overall SCFAs as well as to a highly selective production of specific SCFAs (Fig. 2b). FA output spectra of strains carrying a multiple mutated FAS generally implied a complex and partly non-intuitive behaviour of individual mutational effects. This is, for example, visible on the effect of the I306A mutation on the FA spectrum. The strain carrying solely the I306A mutation did not show an increased yield in SCFAs compared to the wild-type strain, while the insertion of the I306A mutation into the background of a KS double-mutated strain (yielding a I306A-G1250SM1250W-mutated yeast) led to increased $\left(66 \mathrm{mgl}^{-1}\right.$ SCFAs compared to $57 \mathrm{mgl}^{-1}$ for the G1250S-M1250W mutated strain) and the insertion into the background of a R1834K-mutated strain to decreased SCFA production $\left(37 \mathrm{mgl}^{-1}\right.$ SCFAs compared to $100 \mathrm{mgl}^{-1}$ for R1834K; Fig. 2b). In spite of this partly ambiguous impact on overall FA amounts, specific influences of mutations on FA chain length can be derived from our data. Supplementary Table 2 gives an indication on the chainlength regulation effect contributed by a particular mutation.

Within tested strains, some combinations of mutations stand out: The highest total yield in extracellular SCFAs was achieved with the combination of I306A-R1834K-G1250S, producing total amounts of $118 \mathrm{mgl}^{-1}$. For the specific production of $\mathrm{C}_{6}$-FA, the double mutant I306A-G1250S showed best results with $20 \mathrm{mgl}^{-1}$ of $\mathrm{C}_{6}$-FA, accounting for $90 \%$ of the measured SCFAs $\left(\mathrm{C}_{6}\right.$ to $\left.\mathrm{C}_{12}\right)$. Specific production of $\mathrm{C}_{8}$-FA was possible with the triple mutant I306A-R1834K-F1279Y with a titre of $48 \mathrm{mgl}^{-1}$, representing $89 \%$ of its SCFA output.

Vitality parameters of engineered strains. For monitoring growth behaviour, we generally measured wet cell pellet weights 
a
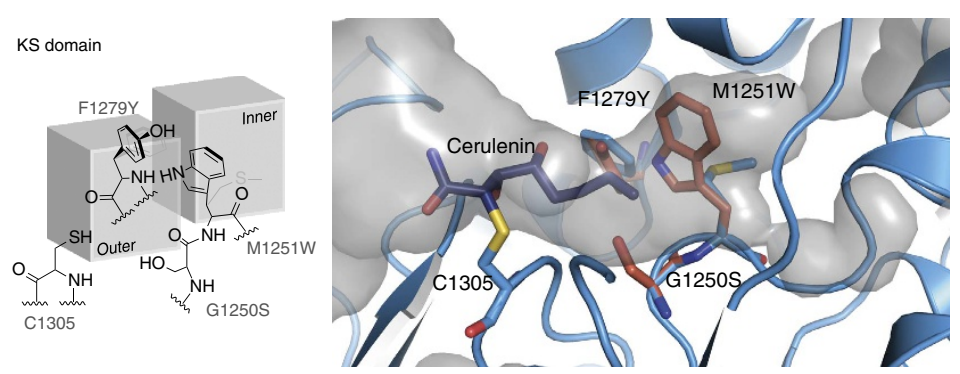

C

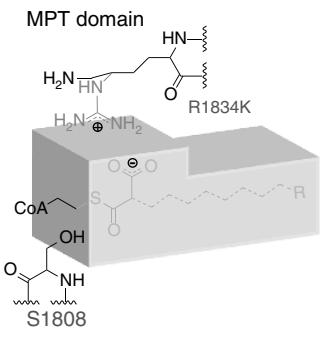

AT domain

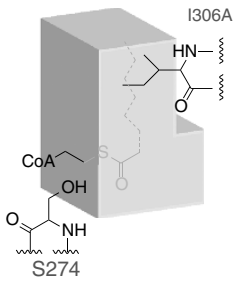

b

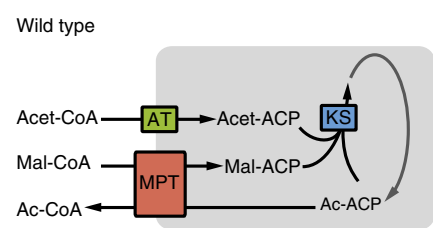

d

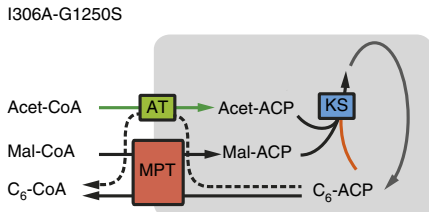

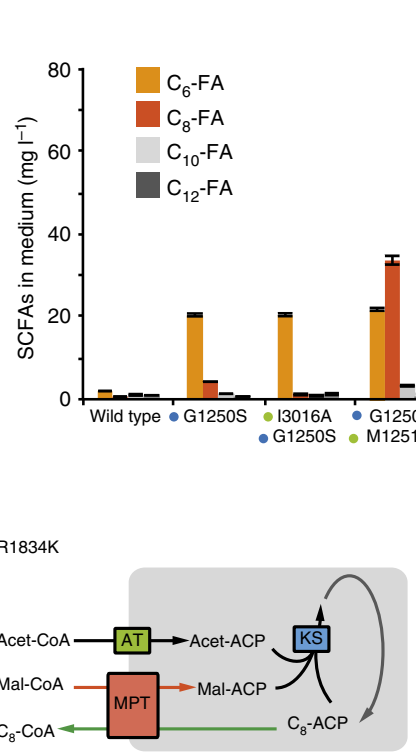

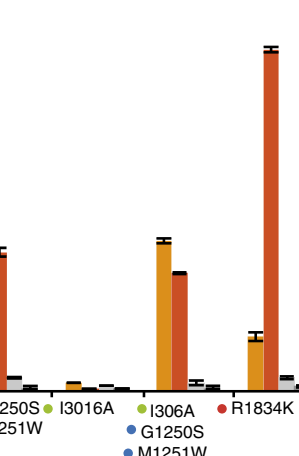

- M1251W
I306A-G1250S-R1834K

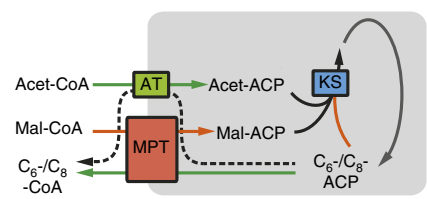

I306A-G1250S-M1251W-R1834K

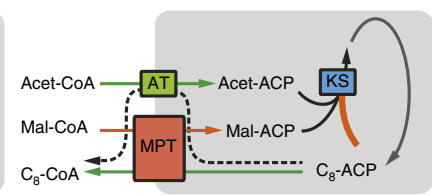

Figure 2 | Active site engineering of S. cerevisiae FAS. (a) KS active site mutated in three positions. (Right panel) X-ray structural data of KS with the binding channel in its open state (structure coloured in blue; PDB code 2VKZ (ref. 19)). Cerulenin, a FAS inhibitor and acyl mimic, which induces binding channel opening, is shown in dark blue. A structural model of G1250S-M1251W-F1279Y-mutated KS in its closed state is superimposed (residues shown in orange). (Left panel) Abstracted KS active site indicating an outer and an inner volume of the acyl binding channel, gated by methionine (native) or tryptophan (mutated FAS), respectively. Amino acids in grey show wild-type KS in its open-state conformation. Bound substrates are omitted for clarity. The closed-state conformation is shown for G1250S-M1251W-F1279Y-mutated KS (residues in black). (b) SCFA product spectra of selected mutated FAS strains in YPD medium. Error bars reflect the standard deviation from three technical replicates (collected on one biological sample). Biological repeatability is shown in Supplementary Fig. 1 and Supplementary Table 1. Please note, while b shows values from one biological sample, values in the text refer to means of two values (Supplementary Table 1). Mutations in KS are indicated by blue dots, and mutations in transferase domains MPT and AT by dots in red and green, respectively. Strains with I306A-R1834K-G1250S-M11251W-mutated FAS and I306A-R1834K-F1279Y-mutated FAS (both marked by \#) grew only to approximately one-third of the regular cell density as compared to the other strains. A scheme on FAS-mediated FA synthesis is attached (flux scheme). Just enzymatic domains are shown that have been mutated in our approach (see for comparison Fig. 1b).

(c) Cartoon representation of AT and MPT active sites. For MPT, the wild-type arginine is shown in grey. Bimodal binding is indicated by substrates with solid and dashed lines; acyl-CoA in dashed lines overlaid with malonyl-CoA in solid lines for MPT, and $\mathrm{C}_{8}$-CoA (dashed) overlaid with acetyl-CoA (solid) for AT. (d) Flux schemes of engineered FASs estimated from data reported in literature, as well as deduced from the extracellular SCFA distribution. Red arrows indicate reduced and green arrows increased fluxes compared to wild type (b). A dashed line indicates a putatively new reaction channel installed by engineering. Flux schemes do not explain relative $C_{6}-/ C_{8}-F A$ levels.

as well as, for selected strains, recorded $\mathrm{OD}_{600}$ at several time points (Supplementary Fig. 2 and Supplementary Table 3). In regular YPD, strains showed growth behaviours ranging from wild-type-like growth rates to extremely low or non-detectable growth rates. We assume that compromised growth relies on the insufficient supply with long FAs from de novo FA synthesis, as resulting from the efficient SCFA production. Supporting this hypothesis, growth defects can be compensated by addition of oleic acid $\left(\mathrm{C}_{18: 1}, 1 \mathrm{mM}\right)$ to the YPD medium (Supplementary Table 3). In YPD medium supplemented with oleic acid, extracellular FA concentrations were changed towards lower yields in SCFAs, with the only exception of the I306A-R1834K-G1250SM1251W-mutated strain producing $131 \mathrm{mgl}^{-1}$ instead of $104 \mathrm{mgl}^{-1}$ of SCFAs (Supplementary Fig. 3). Intriguingly, we observed a shift towards shorter FA, as compared to growth in regular YPD, with the G1250S-M1251W-mutated strain as most prominent example. It is tempting to speculate that this effect is based on an inhibition of acetyl-CoA carboxylase Acc1 (ref. 35), leading to lower malonyl-CoA levels and shorter FAs ${ }^{33,34}$. However, other more complex regulatory effects by long-chain FA, as for example on the FA cycle or on upstream enzymes, may be involved ${ }^{35}$.

Regarding a putative impact on growth by toxic SCFAs ${ }^{36,37}$, we were interested in clarifying whether a strong initial production of $\mathrm{C}_{8}$-FA accounts for the inhibition of further growth. Product spectra of selected strains were recorded after 12, 24 and $48 \mathrm{~h}$; the latter constituting the regularly chosen measurement point. While strains showed varying growth rates (Supplementary Fig. 2), the FA production profile was largely conserved reaching a maximum yield in SCFAs after $48 \mathrm{~h}$ (Supplementary Fig. 4). These data 
argue against an early growth limitation of some strains by high inhibitory concentration of SCFAs. Glucose consumption and ethanol levels were recorded during the cultivations. All tested strains behaved similarly with glucose being entirely consumed after about $20 \mathrm{~h}$, followed by a partial consumption of the produced ethanol (Supplementary Fig. 5).

Effect of promoter exchange on FA synthesis. For the production of SCFAs in S. cerevisiae, FAS2 and FAS1 were generally under control of their natural promoters and terminators ${ }^{22}$. We favoured this approach, as it is minimal-invasive and allows comparison with wild-type output spectra prior to applying means for FA overproduction ${ }^{7}$. To further evaluate the potential of our mutations, we performed a single layer of optimization, and probed FA production under FAS overexpression conditions. We therefore complemented the $\Delta$ fas $1 \Delta$ fas 2 strain with vectors, in which genes FAS2 and FAS1 are put under control of the alcohol dehydrogenase II promoter of S. cerevisiae ( $p A D H 2)$. We worked with pRS- $p A D H 2$ vectors to generate R1834K-mutated ( $\mathrm{C}_{8}$-FA producing), I306A-G1250S-double mutated $\left(\mathrm{C}_{6}\right.$-FA) and I306A-G1250S-R1834K-triple mutated $\left(\mathrm{C}_{6}\right.$-FA and $\mathrm{C}_{8}$-FA) yeast strains. As $p A D H 2$ is repressed in the presence of glucose ${ }^{38}$, and we have observed the consumption of glucose after about $20 \mathrm{~h}$ (Supplementary Fig. 5), we expected highest extracellular FA levels at longer cultivaton times. In addition, we buffered the medium by supplementing YPD medium with $250 \mathrm{mM}$ potassium phosphate. Under these conditions, we observed a substantial increase in yields, with concentrations of $\mathrm{C}_{8}$-FA of $245.0 \mathrm{mgl}^{-1}$ (R1834K; $85.9 \%$ of total extracellular SCFAs), $\mathrm{C}_{6}$-FA of $71.5 \mathrm{mgl}^{-1}$ (I306A-G1250S; 78.2\%), and mixed $\mathrm{C}_{6}$-FA/C - -FA of $464.4 \mathrm{mgl}^{-1}$ (I306A-G1250S-R1834K, 96.0\%; Fig. 3a), which corresponds to the highest titre of SCFAs reported for yeast to date.

The origin of short-chain acyl-CoA thioesterase activity. Recently, Eht1 was described to act as octanoyl-CoA:ethanol acyltransferase and as thioesterase ${ }^{39}$. Assuming that the whole family of short-chain AEATases, comprising Eht1 (open reading frames YBR177C), Eeb1 (YPL095C) and Mgl2 (YMR210W), share this dual activity ${ }^{40}$, we generated a set of strains deficient in candidate proteins to identify the origin of the hydrolysing activity, which processes short-chain acyl-CoAs to SCFAs that are finally exported to the culture medium. Specifically, we constructed strains with the single genes deleted ( $\Delta e h t 1, \Delta e e b 1$ and $\Delta m g l 2)$, with deletions in two genes (Aeeb1 $\Delta e h t 1, \Delta e h t 1$ $\Delta m g l 2)$ and with all three genes deleted (Aeeb1 $\Delta e h t 1 \quad \Delta m g l 2)$. All strains were also deficient in FAS ( $\Delta$ fas $1 \Delta$ fas2). Working with this set of strains, complemented with engineered FAS, allowed addressing the question, whether the observed hydrolysis is accounted by a specific AEATase or by all three candidate AEATases.

The single deletions had a minor impact on extracellular SCFA levels, ruling out that a single AEATase is responsible for acyl-CoA hydrolysis. Data also demonstrate that upstream effects interfering in this study are unlikely (that is, deletions acting on FAS substrate levels, or deletions imposing indirect effects as for example regulatory effects). The two double-knockout strains generally caused reduced extracellular SCFA levels (particularly $\Delta e e b 1$ Aeht 1 ), while finally the triple knockout ( $\Delta e e b 1 \Delta e h t 1 \Delta m g l 2)$ led to essentially abolished production of SCFAs (Fig. 3b), suggesting that all three AEATases are responsible for short-chain acyl-CoA hydrolysis in SCFA producing strains.

Interestingly, AEATase deletions had different effects on FAS mutants. For both FAS mutants, R1834K and I306A-
R1834K-G1250S, we found varying FA product distributions in AEATase single-knockout strains. In line with the reported specificity of Eht1 for $\mathrm{C}_{8}$-CoA, we measured a significant drop of $\mathrm{C}_{8}$-FA concentrations in the Eht1-deficient strain $^{39}$. Similarly, our data suggest that Eeb1 has higher selectivity for $\mathrm{C}_{6}$-CoA. Data of the double AEATase knockout strains support the specificities suggested from single knockouts (Fig. 3b). While the detailed molecular basis for the interplay of FAS and AEATases remains elusive, the observed phenomena are important per se. In our approach, which is powerful in producing SCFAs in narrow chain-length distributions, thiosterases (as AEATases) might impose a valuable downstream selectivity filter that can further improve purity and yield of free FA products.

\section{Discussion}

The biosynthetic potential of nature for the processing of materials had been exploited for centuries, when the discipline biotechnology put an originally phenomenologically driven practice onto scientific grounds. Since then, the broad and efficient production of a large variety of compounds has been achieved, continuously replacing and complementing traditional synthetic chemical strategies. In spite of all progress, the accessible synthetic spectrum of products is still considerably restricted by the native function of the cellular components involved in the biosynthetic processes. Developing biotechnology to a versatile synthetic tool requires the modulation of specificities of natural systems to enlarge the spectrum of biotechnologically accessible compounds. Likewise, it is one of the foremost goals of bioengineering approaches, that aim at expanding the product portfolio, to conserve or even improve the intrinsic efficiency of natural systems for warranting high product yields ${ }^{41}$.

In the presented study, we describe the minimally invasive engineering of S. cerevisiae FAS for the construction of SCFAs producing yeast strains. We identified a set of only five mutations that are capable of significantly controlling FA chain-length regulation. By applying mutations in several combinations, an overall titre of SCFAs of $464.4 \mathrm{mgl}^{-1}$ was achieved, which corresponds to a 109-fold increase over wild type, as well as the selective production of FAs of specific chain lengths $\left(\mathrm{C}_{6}\right.$-FA: $71.5 \mathrm{mgl}^{-1}, 78.2 \%$ of total extracellular SCFAs; $\mathrm{C}_{8}$-FA: $245.0 \mathrm{mgl}^{-1}, 85.9 \%$; Fig. $3 \mathrm{a}$ ). The successful approach redirected kinetic fluxes of de novo FA synthesis by modulating substrate specificities of FAS enzymatic domains, while keeping the integrity of the protein intact. Mutations act directly on chainlength control, by interfering in the elongation of long acyl chains (KS mutations: G1250S, M1251W, F1279Y; Fig. 2a), as well as by favouring the release of short acyl chains through a weakened binding of the competing malonyl (MPT mutation R1834K) and a broadened acetyl binding channel (AT mutation I306A; Fig. 2c). In addition, mutations act indirectly on chain-length control by increasing acetyl/malonyl ratios, which pronounce priming of fatty acid synthesis and shift the spectrum towards shorter FA. By this approach, we intended to steer FA synthesis towards the premature release of the short acyl-chain without evoking a drop in synthetic rates. Among the mutations employed in FAS engineering, the MPT mutation is, however, clearly a costly one. It reduces the uptake of the malonyl moiety required for every new elongation cycle and likely affects turnover numbers. As a call for a first improvement of the presented system, FAS engineering needs to be optimized towards increased affinities of MPT for short-chain acyl-CoAs to increase acyl export rates without decreasing malonyl import.

Although specific information on the direct product spectra of FAS mutants is missing, we assume a bimodal chain-length 
a
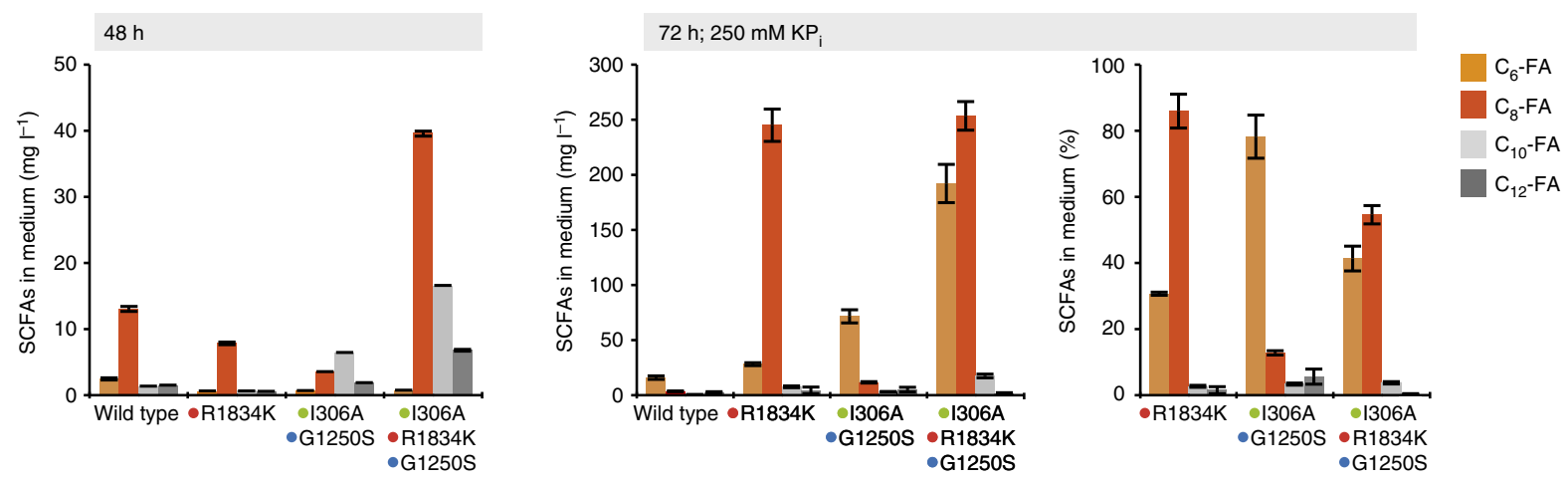

b

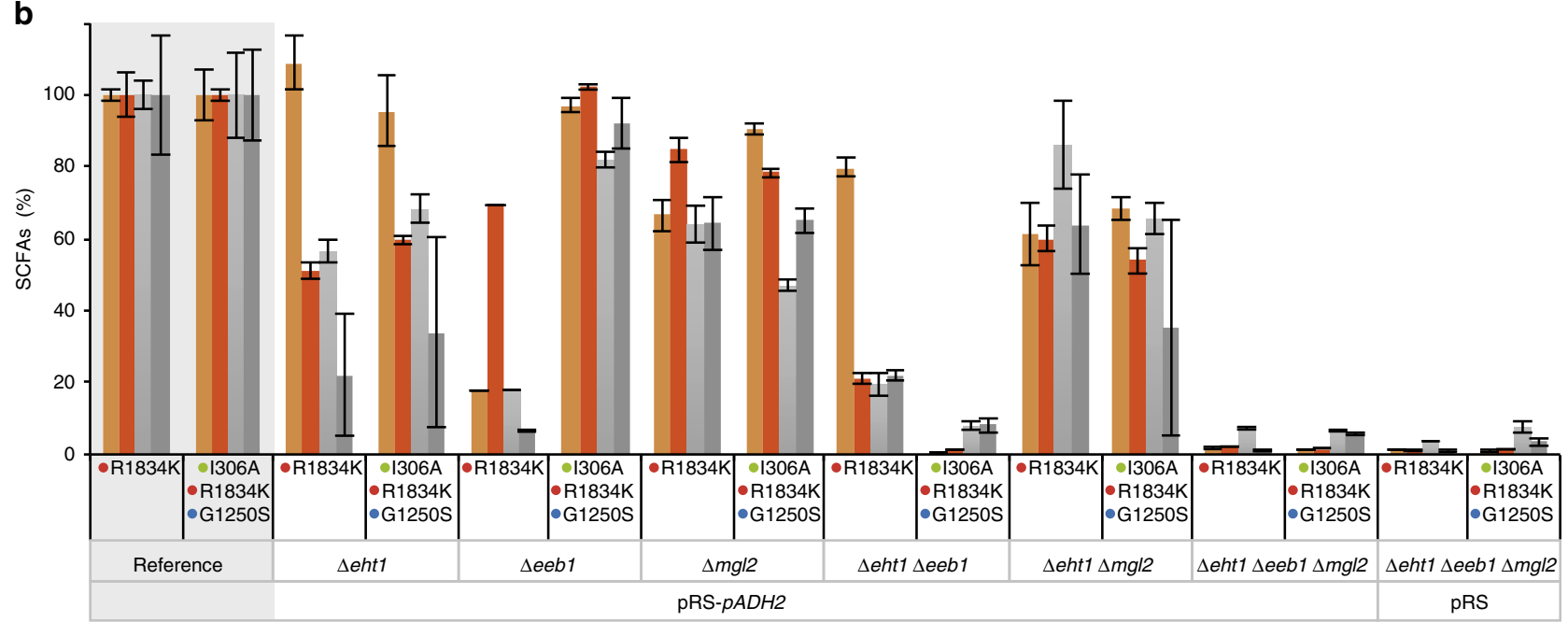

Figure 3 | SCFA levels in FAS overproducing strains and AEATase knockout strains. (a) Product spectra of S. cerevisiae $\Delta$ fas 14 fas 2 complemented with selected FAS expressed from $p A D H 2$ promoter-controlled genes. Expression conditions as described in Fig. $2 \mathrm{~b}$, with cultures grown for $48 \mathrm{~h}$ in non-buffered and $72 \mathrm{~h}$ in buffered medium ( $250 \mathrm{mM}$ potassium phosphate, $\mathrm{KP}$ ). Experiments were performed with statistics as described in Fig. $2 \mathrm{~b}$. For cultures grown for $72 \mathrm{~h}$, product distribution is also given in shares of total yields. (b) Percentage of extracellular FAs detected in medium in AEATase knockout strains. All AEATase knockout strains are also deficient in FAS ( $\triangle$ fas $1 \Delta$ fas2; not indicated by figure labels). Strains are complemented with SCFA producing FAS mutants, as indicated, with genes under control of the wild type and the $\mathrm{pADH} 2$ promoter. Recorded extracellular SCFA concentrations are given in percentage and relate to reference ( $\triangle$ fas 1 ffas 2 ), which is set to $100 \%$ (grey background). Cultivation was performed for $72 \mathrm{~h}$ in $100 \mathrm{mM}$ buffered phosphate medium. Experiments were performed with statistics as described in Fig. $2 b$.

distribution of products composed of short-chain acyl-CoAs, which are finally exported as free FAs to the medium, and normal length $\mathrm{C}_{16^{-}}$and $\mathrm{C}_{18^{-}} \mathrm{CoA}$. This assumption is based on data that were collected on cerulenin derivatives modulated in the length of their aliphatic moiety (for cerulenin binding to KS, see Fig. 2a). These derivatives showed a bimodal profile in inhibition strength, indicating weak binding of compounds of medium length ${ }^{42}$. A bimodal product spectrum of FAS variants may be explained in a similar manner as originating from KS engineering. Short-chain acyl-CoA esters are produced, as the respective acyl chains are hindered in KS binding by clashing with the gate keeper residue. Long-chain acyl-CoA esters result from acyl chains that escape early release and grow into the inner volume of the KS-binding channel, where they finally underlie constraints of original chainlength control (Fig. 2a). Such a leaky chain-length control can explain the viability of most of the FAS-mutated strains, as well as the growth deficit in FAS variants with accumulated critical mutations (see Supplementary Fig. 2 and Supplementary Table 3).

In our study, we have further identified three AEATases (Eeb1, Eht1 and Mgl2) that are responsible for the hydrolysis of short-chain acyl-CoA esters ${ }^{39,40}$. In a combinatorial approach, generating a set of AEATase-deficient strains, we were able to reveal the collaboration of all three proteins for hydrolysis (Fig. 3b). AEATases, as well as thioesterases in general, can be valuable tools when assisting de novo chain-length control. For example, for the putative task of the specific production of $\mathrm{C}_{8}$-FA, the Eeb1-deficient strain might be the better suited host for R1834K-mutated FAS than the wild-type strain, as it leads to higher product purity (Fig. 3b). In such combined approaches, recombinant thioesterases with narrow product spectra might be even more powerful ${ }^{23}$. AEATases and thioesterases can also be useful in an inverse setting. Short-chain acyl-CoAs produced with the presented FAS mutants might be ultra-specifically directed into downstream biosynthetic pathways processes, when clearing the cytoplasm from unwanted acyl-CoAs by selective thioesterase activity $4,43,44$.

Our study on structure-based rational mutagenesis of S. cerevisiae FAS represents a novel minimally invasive approach for chain-length control in de novo FA synthesis. We succeeded in generating a set of mutations that is capable of inducing SCFA production by redirecting kinetic fluxes in de novo FA synthesis by FAS. The presented system can be seen as a module that can turn on SCFA production in essentially any microbial organism. With the advantage of gaining access 
to CoA esters, as the immediate products of fungal FA synthesis, this technology is also highly attractive for biosynthetic processes that aim at the synthesis of FA derived substances, for example, as alkanes and alcohols for biofuel production ${ }^{4,45}$, or at providing acyl-CoAs as starter compound for other biosynthetic clusters ${ }^{43}$.

\section{Methods}

Description yeast strain. The haploid S. cerevisiae strain BY.PK1238_1A_KO, which was kindly provided by Peter Kötter (University of Frankfurt, Germany) and used in this work, has a BY background and the reading frames of FAS1 and FAS2 are each replaced by a kanMX4 cassette, resulting in a clean knockout of FAS and antibiotic resistance against geneticin. The exact genotype is Mat $\alpha$; ura3 $\triangle 0$; his3 $\triangle 0$; leu2 $\triangle 0$; TRP1; lys2 00 ; MET15; fas 1::uptag-kanMX4-downtag; fas2::uptag-kanMX4-downtag. The deletion of three thioesterase genes (EEB1, EHT1 and MGL2) was performed using the simplified CRISPR-Cas genome editing tool for S. cerevisiae ${ }^{46}$

Vector description. The vectors used in this work are centromeric pRS shuttle vectors of types pRS313 and pRS315 (ref. 47) with single copy number and HIS3 and LEU2 auxotrophy marker, respectively. FAS1 or mutations thereof were always provided on pRS315, while FAS2 or mutations thereof were always provided on pRS313, each regulated by its corresponding native promoter ( 995 bp upstream for FAS1 and 480 bp upstream for FAS2 ${ }^{22}$. Terminator sequences were set to $295 \mathrm{bp}$ and $258 \mathrm{bp}$, respectively, downstream of the open reading frames. Cloning was always done in E. coli using the Infusion HD cloning kit (Clontech, Mountain View, USA). Wild-type FAS1 and FAS2 genes were assembled from several fragments, which were amplified from S. cerevisiae genomic DNA, into pRS vectors using BamHI and SalI restriction sites. Exact chromosomal coordinates including promoter and terminator sequences according to strain S288C are for FAS1 (YKL182w): Chr XI 99676-107121 and for FAS2 (YPL231w): ChrXVI 108172114573. In addition to pRS-based expression, FAS encoding genes FAS1 and FAS2, in wild-type and mutated variants, were also provided under control of the S. cerevisiae alcohol dehydrogenase 2 promoter (ADH2; $573 \mathrm{bp}$ ). Vectors are similar to pRS-based vectors, described above, except that the promoter has been exchanged. Vectors are termed pRS-pADH2. Primers for site-directed mutagenesis are listed in Supplementary Table 4.

Transformation. For yeast transformations, $\sim 1 \mu \mathrm{g}$ of each plasmid DNA was co-transformed following a modified lithium acetate protocol ${ }^{48}$. A $5 \mathrm{ml}$ overnight culture of strain BY.PK1238_1A_KO in YPD (1\% yeast extract, $2 \%$ peptone, both produced by BD, Difco Laboratories, Sparks, USA; $2 \%$ dextrose, purchased from Roth, Karlsruhe, Germany) containing $200 \mu \mathrm{g} \mathrm{ml}^{-1}$ geneticin disulfate, free FAs (myristic, palmitic and stearic acid, each $50 \mu \mathrm{g} \mathrm{ml}^{-1}$ ) and $1 \%$ Tween20 grown at $30^{\circ} \mathrm{C}$ and 200 r.p.m. was used to inoculate a main culture in the same medium. After shaking at $30^{\circ} \mathrm{C}$ and 200 r.p.m. to $\mathrm{OD}_{600}=0.8$, a volume of $5 \mathrm{ml}$ of this culture was harvested by centrifugation $\left(3,000\right.$ r.c.f., $\left.5 \mathrm{~min}, 24^{\circ} \mathrm{C}\right)$. The cells were washed by resuspending in $1 \mathrm{ml}$ water and centrifuged again. After resuspension in lithium acetate solution $(0.1 \mathrm{M})$, cells were incubated for $5 \mathrm{~min}$ at $24^{\circ} \mathrm{C}$ and centrifuged $\left(5,000\right.$ r.c.f., $\left.15 \mathrm{~s}, 24^{\circ} \mathrm{C}\right)$, before the transformation mix was added ( $240 \mu \mathrm{l}$ PEG 1,500 solution (50\%), $76 \mu \mathrm{l}$ water, $36 \mu \mathrm{l}$ lithium acetate solution $(1.0 \mathrm{M}), 5.0 \mu \mathrm{l}$ single-stranded DNA solution from salmon testis $\left(10 \mathrm{mg} \mathrm{ml}^{-1}\right), 2 \mu \mathrm{l}$ of each plasmid DNA solution). The cell suspension was mixed well and incubated for $30 \mathrm{~min}$ at $30^{\circ} \mathrm{C}$ followed by $20 \mathrm{~min}$ at $42^{\circ} \mathrm{C}$. After pelleting the cells by centrifugation $\left(4,000\right.$ r.c.f., $\left.15 \mathrm{~s}, 4^{\circ} \mathrm{C}\right)$, they were washed with $1 \mathrm{ml}$ water, pelleted again $\left(4,000\right.$ r.c.f., $\left.15 \mathrm{~s}, 4^{\circ} \mathrm{C}\right)$ and resuspended in $100 \mu \mathrm{l}$ water. For selection of the yeast transformants, the cell suspension was spread on SCD-his-leu agar plates containing $200 \mu \mathrm{g} \mathrm{ml}^{-1}$ geneticin disulfate, free FAs (myristic, palmitic and stearic acid, each $50 \mu \mathrm{g} \mathrm{ml}^{-1}$ ) and $1 \%$ Tween 20 .

Cultures for product analysis. S. cerevisiae strains were cultured in YPD medium (composition as described above). For cultivation of FAS encoded by genes under the $p A D H 2$ promoter for 72 and $96 \mathrm{~h}$, the medium was additionally buffered with 100 or $250 \mathrm{mM}$ potassium phosphate (as indicated in figure legends). For product analyses, several colonies of the $S$. cerevisiae strains were picked and combined in one pre-culture (5 ml YPD with $200 \mu \mathrm{g} \mathrm{ml}^{-1}$ geneticin disulfate, $50 \mathrm{mg} \mathrm{ml}^{-1}$ final concentration). After shaking at 200 r.p.m. at $30^{\circ} \mathrm{C}$ over night, the $\mathrm{OD}_{600}$ was measured. The main culture $\left(50 \mathrm{ml}\right.$ YPD with $200 \mu \mathrm{g} \mathrm{ml}^{-1}$ geneticin disulfate, $50 \mathrm{mg} \mathrm{ml}^{-1}$ final concentration) was inoculated to $\mathrm{OD}_{600}=0.1$ and shaken for $48 \mathrm{~h}$ at 200 r.p.m. and $30^{\circ} \mathrm{C}$. Before further processing, the $\mathrm{OD}_{600}$ was measured again. For samples with long FAs supplementation, $\mathrm{C}_{18: 1}$ and Tergitol NP-40 (solution in water, $70 \%$ ) were added to all cultures to a final concentration of $1 \mathrm{mM}$ or $1 \%$ in the case of Tergitol. Buffered medium was used for strains with FAS encoding genes under the $\mathrm{pADH} 2$ promoter.

Sample processing. FA extraction was performed as follows ${ }^{23}$ : first cells were spun down at 3,500 r.c.f. for $15 \mathrm{~min}$. The supernatant was aliquoted in $10 \mathrm{ml}$ portions and $0.2 \mathrm{mg}$ of the internal standard, heptanoic acid $\left(\mathrm{C}_{7}\right)$, dissolved in
$100 \mu \mathrm{l}$ of solvent (33\% methanol, $67 \%$ chloroform) was added. After acidification with $1 \mathrm{ml} \mathrm{HCl}(1 \mathrm{M}), 2.5 \mathrm{ml}$ of a mixture of equal amounts of methanol and chloroform were added. The samples were shaken vigorously for $5 \mathrm{~min}$ and then centrifuged again at 3,500 r.c.f. for $10 \mathrm{~min}$. The chloroform layer was transferred to a new vial and any residual water removed. The liquid was then fully evaporated in a SpeedVac. For methylation, $0.3 \mathrm{ml}$ of $\mathrm{HCl}(8 \%$ solution made from $9.7 \mathrm{ml}$ of concentrated $\mathrm{HCl}$ and $41.5 \mathrm{ml}$ of methanol), $0.2 \mathrm{ml}$ of toluene and $1.5 \mathrm{ml}$ of methanol were added for resuspension ${ }^{49}$. The mixture was vortexed and later heated to $100^{\circ} \mathrm{C}$ for $3 \mathrm{~h}$. After a cool down on ice, $1 \mathrm{ml}$ hexane and $1 \mathrm{ml}$ water were added. The samples were vortexed once again and after separation, the organic layer was transferred in a vial for further analysis by gas chromatography (GC).

Determination of free FAs by GC. The resulting FA methyl esters (dissolved in hexane) were measured with a Perkin Elmer Clarus 400 gas chromatograph (Perkin Elmer, Rodgau, Germany) equipped with an Elite FFAP capillary column $(30 \mathrm{~m} \times 0.25 \mathrm{~mm}$, film thickness: $0.25 \mu \mathrm{m}$; Perkin Elmer, Rodgau, Germany) and a flame ionization detector (Perkin Elmer, Rodgau, Germany). A volume of $1 \mu \mathrm{l}$ of the sample was analysed after split injection $\left(10 \mathrm{ml} \mathrm{min}^{-1}\right)$ and helium as carrier gas. The temperatures of the injector and detector were 200 and $250^{\circ} \mathrm{C}$, respectively. The following temperature program was applied: $50^{\circ} \mathrm{C}$ for $5 \mathrm{~min}$, increase of $10^{\circ} \mathrm{C} \mathrm{min}^{-1}$ to $120^{\circ} \mathrm{C}$ (hold for $5 \mathrm{~min}$ ), increase of $15^{\circ} \mathrm{C} \mathrm{min}-1$ to $180^{\circ} \mathrm{C}$ (hold for $10 \mathrm{~min}$ ), and increase of $20^{\circ} \mathrm{C} \mathrm{min}-1$ to $220^{\circ} \mathrm{C}$ for $37 \mathrm{~min}$.

For each culture, three $10 \mathrm{ml}$ aliquots from the same media were separately processed and measured by GC. The standard deviation between the three measurements from the same media was typically below $1 \mathrm{mgl}^{-1}$ (for $92 \%$ of the measured concentrations).

Metabolite analysis by HPLC. For quantification of glucose and ethanol, $450 \mu \mathrm{l}$ cell-free samples were mixed with $50 \mu \mathrm{l}$ of $50 \%$ (w/v) 5-sulfosalicylic acid, vigorously shaken and centrifuged $\left(4^{\circ} \mathrm{C}, 5 \mathrm{~min}, 13,000\right.$ r.c.f.). The supernatant was analysed with an UHPLC + system (Dionex UltiMate 3000, Thermo Scientific, USA) equipped with a HyperREZ XP Carbohydrate $\mathrm{H}+8 \mu \mathrm{m}$ column. To detect the substrates, a refractive index detector (Thermo Shodex RI-101) was used. Separation was carried out at $65^{\circ} \mathrm{C}$ with $5 \mathrm{mM}$ sulfuric acid as mobile phase (flow rate of $0.6 \mathrm{ml} \mathrm{min}^{-1}$ ). Five standards (mixtures of D-glucose, ethanol, glycerol and acetate with concentrations of $0.05-2 \%(w / v))$ were analysed for quantification of the different compounds.

Determination of cell density. The cell density in a liquid culture was measured with an Ultrospec 2100 pro spectrophotometer (GE Healthcare, USA) by determination of the optical density at $600 \mathrm{~nm}\left(\mathrm{OD}_{600}\right)$.

Data availability. All data generated or analysed during this study are included in this published article (and its Supplementary Information file) or are available from the corresponding author upon reasonable request.

\section{References}

1. Schweizer, E. \& Hofmann, J. Microbial type I fatty acid synthases (FAS): major players in a network of cellular FAS systems. Microbiol. Mol. Biol. Rev. 68, 501-517 (2004).

2. Tehlivets, O., Scheuringer, K. \& Kohlwein, S. D. Fatty acid synthesis and elongation in yeast. Biochim. Biophys. Acta 1771, 255-270 (2007).

3. Runguphan, W. \& Keasling, J. D. Metabolic engineering of Saccharomyces cerevisiae for production of fatty acid-derived biofuels and chemicals. Metab. Eng. 21, 103-113 (2014).

4. Choi, Y. J. \& Lee, S. Y. Microbial production of short-chain alkanes. Nature 502, 571-574 (2013).

5. Leber, C., Polson, B., Fernandez-Moya, R. \& Da Silva, N. A. Overproduction and secretion of free fatty acids through disrupted neutral lipid recycle in Saccharomyces cerevisiae. Metab. Eng. 28, 54-62 (2015).

6. Zhou, Y. J. et al. Production of fatty acid-derived oleochemicals and biofuels by synthetic yeast cell factories. Nat. Commun. 7, 11709 (2016).

7. Zhang, F. et al. Enhancing fatty acid production by the expression of the regulatory transcription factor FadR. Metab. Eng. 14, 653-660 (2012).

8. Peralta-Yahya, P. P., Zhang, F., del Cardayre, S. B. \& Keasling, J. D. Microbial engineering for the production of advanced biofuels. Nature 488, 320-328 (2012).

9. Xu, P., Qiao, K., Ahn, W. S. \& Stephanopoulos, G. Engineering Yarrowia lipolytica as a platform for synthesis of drop-in transportation fuels and oleochemicals. Proc. Natl Acad. Sci. USA 113, 10848-10853 (2016).

10. Fichtlscherer, F., Wellein, C., Mittag, M. \& Schweizer, E. A novel function of yeast fatty acid synthase. Subunit alpha is capable of self-pantetheinylation. Eur. J. Biochem. 267, 2666-2671 (2000).

11. Stuible, H. P., Meurer, G. \& Schweizer, E. Heterologous expression and biochemical characterization of two functionally different type I fatty acid synthases from Brevibacterium ammoniagenes. Eur. J. Biochem. 247, 268-273 (1997). 
12. Jayakumar, A. et al. Human fatty acid synthase: properties and molecular cloning. Proc. Natl Acad. Sci. USA 92, 8695-8699 (1995).

13. Anselmi, C., Grininger, M., Gipson, P. \& Faraldo-Gomez, J. Mechanism of substrate shuttling by the acyl-carrier protein within the fatty acid megasynthase. J. Am. Chem. Soc. 132, 12357-12364 (2010).

14. Gipson, P. et al. Direct structural insight into the substrate-shuttling mechanism of yeast fatty acid synthase by electron cryomicroscopy. Proc. Natl Acad. Sci. USA 107, 9164-9169 (2010).

15. Beld, J., Lee, D. J. \& Burkart, M. D. Fatty acid biosynthesis revisited: structure elucidation and metabolic engineering. Mol. Biosyst. 11, 38-59 (2015).

16. Leibundgut, M., Jenni, S., Frick, C. \& Ban, N. Structural basis for substrate delivery by acyl carrier protein in the yeast fatty acid synthase. Science 316, 288-290 (2007).

17. Jenni, S. et al. Structure of fungal fatty acid synthase and implications for iterative substrate shuttling. Science 316, 254-261 (2007).

18. Lomakin, I. B., Xiong, Y. \& Steitz, T. A. The crystal structure of yeast fatty acid synthase, a cellular machine with eight active sites working together. Cell 129, 319-332 (2007).

19. Johansson, P. et al. Inhibition of the fungal fatty acid synthase type I multienzyme complex. Proc. Natl Acad. Sci. USA 105, 12803-12808 (2008).

20. Maier, T., Leibundgut, M., Boehringer, D. \& Ban, N. Structure and function of eukaryotic fatty acid synthases. Q. Rev. Biophys. 43, 373-422 (2010).

21. Grininger, M. Perspectives on the evolution, assembly and conformational dynamics of fatty acid synthase type I (FAS I) systems. Curr. Opin. Struct. Biol. 25, 49-56 (2014).

22. Chirala, S. S. Coordinated regulation and inositol-mediated and fatty acidmediated repression of fatty acid synthase genes in Saccharomyces cerevisiae. Proc. Natl Acad. Sci. USA 89, 10232-10236 (1992).

23. Leber, C. \& Da Silva, N. A. Engineering of Saccharomyces cerevisiae for the synthesis of short chain fatty acids. Biotechnol. Bioeng. 111, 347-358 (2014).

24. Olsen, J. G., Kadziola, A., von Wettstein-Knowles, P., Siggaard-Andersen, M. \& Larsen, S. Structures of beta-ketoacyl-acyl carrier protein synthase I complexed with fatty acids elucidate its catalytic machinery. Structure 9, 233-243 (2001).

25. Christensen, C. E., Kragelund, B. B., Von Wettstein-Knowles, P. \& Henriksen, A. Structure of the human beta -ketoacyl $[\mathrm{ACP}]$ synthase from the mitochondrial type II fatty acid synthase. Protein Sci. 16, 261-272 (2007).

26. Aritomi, K. et al. Self-cloning yeast strains containing novel FAS2 mutations produce a higher amount of ethyl caproate in Japanese sake. Biosci. Biotechnol. Biochem. 68, 206-214 (2004).

27. Hitchman, T. S. et al. Hexanoate synthase, a specialized type I fatty acid synthase in aflatoxin B1 biosynthesis. Bioorg. Chem. 29, 293-307 (2001).

28. Woloshuk, C. P. \& Prieto, R. Genetic organization and function of the aflatoxin B1 biosynthetic genes. FEMS Microbiol. Lett. 160, 169-176 (1998).

29. Engeser, H., Hubner, K., Straub, J. \& Lynen, F. Identity of malonyl and palmitoyl transferase of fatty acid synthetase from yeast. 2. A comparison of active-site peptides. Eur. J. Biochem. 101, 413-422 (1979).

30. Pirson, W., Schuhmann, L. \& Lynen, F. Specificity of yeast fatty acid synthetase with respect to the priming substrate. Decanoyl-CoA and derivatives as primers of fatty acid synthesis in vitro. Eur. J. Biochem. 36, 16-24 (1973).

31. Bunkoczi, G. et al. Structural basis for different specificities of acyltransferases associated with the human cytosolic and mitochondrial fatty acid synthases. Chem. Biol. 16, 667-675 (2009).

32. Rangan, V. S. \& Smith, S. Alteration of the substrate specificity of the malonylCoA/acetyl-CoA:acyl carrier protein S-acyltransferase domain of the multifunctional fatty acid synthase by mutation of a single arginine residue. J. Biol. Chem. 272, 11975-11978 (1997).

33. Sumper, M., Oesterhelt, D., Riepertinger, C. \& Lynen, F. Synthesis of various carboxylic acids by the multienzyme complex of fatty acid synthesis from yeast, and clarification of their structure. Eur. J. Biochem. 10, 377-387 (1969).

34. Kawaguchi, A., Arai, K., Seyama, Y., Yamakawa, T. \& Okuda, S. Substrate control of termination of fatty acid biosynthesis by fatty acid synthetase from Brevibacterium ammoniagenes. J. Biochem. 88, 303-306 (1980).

35. Ogiwara, H., Tanabe, T., Nikawa, J. \& Numa, S. Inhibition of rat-liver acetylcoenzyme-A carboxylase by palmitoyl-coenzyme A. Formation of equimolar enzyme-inhibitor complex. Eur. J. Biochem. 89, 33-41 (1978).

36. Borrull, A., Lopez-Martinez, G., Poblet, M., Cordero-Otero, R. \& Rozes, N. New insights into the toxicity mechanism of octanoic and decanoic acids on Saccharomyces cerevisiae. Yeast 32, 451-460 (2015).

37. Liu, P. et al. Membrane stress caused by octanoic acid in Saccharomyces cerevisiae. Appl. Microbiol. Biotechnol. 97, 3239-3251 (2013).

38. Gancedo, J. M. Yeast carbon catabolite repression. Microbiol. Mol. Biol. Rev. 62, 334-361 (1998).

39. Knight, M. J., Bull, I. D. \& Curnow, P. The yeast enzyme Eht1 is an octanoylCoA:ethanol acyltransferase that also functions as a thioesterase. Yeast 31, 463-474 (2014).
40. Saerens, S. M. et al. The Saccharomyces cerevisiae EHT1 and EEB1 genes encode novel enzymes with medium-chain fatty acid ethyl ester synthesis and hydrolysis capacity. J. Biol. Chem. 281, 4446-4456 (2006).

41. Singh, R. K., Tiwari, M. K., Singh, R. \& Lee, J. K. From protein engineering to immobilization: promising strategies for the upgrade of industrial enzymes. Int. J. Mol. Sci. 14, 1232-1277 (2013).

42. Morisaki, N. et al. Effect of side-chain structure on inhibition of yeast fatty-acid synthase by cerulenin analogs. Eur. J. Biochem. 211, 111-115 (1993).

43. Gagne, S. J. et al. Identification of olivetolic acid cyclase from Cannabis sativa reveals a unique catalytic route to plant polyketides. Proc. Natl Acad. Sci. USA 109, 12811-12816 (2012).

44. d'Espaux, L., Mendez-Perez, D., Li, R. \& Keasling, J. D. Synthetic biology for microbial production of lipid-based biofuels. Curr. Opin. Struct. Biol. 29, 58-65 (2015).

45. Kung, Y., Runguphan, W. \& Keasling, J. D. From fields to fuels: recent advances in the microbial production of biofuels. ACS Synth. Biol. 1, 498-513 (2012).

46. Generoso, W. C., Gottardi, M., Oreb, M. \& Boles, E. Simplified CRISPR-Cas genome editing for Saccharomyces cerevisiae. J. Microbiol. Methods 127, 203-205 (2016).

47. Sikorski, R. S. \& Hieter, P. A system of shuttle vectors and yeast host strains designed for efficient manipulation of DNA in Saccharomyces cerevisiae. Genetics 122, 19-27 (1989).

48. Schiestl, R. H. \& Gietz, R. D. High efficiency transformation of intact yeast cells using single stranded nucleic acids as a carrier. Curr. Genet. 16, 339-346 (1989).

49. Ichihara, K. \& Fukubayashi, Y. Preparation of fatty acid methyl esters for gas-liquid chromatography. J. Lipid Res. 51, 635-640 (2010).

50. Johansson, P. et al. Multimeric options for the auto-activation of the Saccharomyces cerevisiae FAS type I megasynthase. Structure 17, 1063-1074 (2009).

\section{Acknowledgements}

We thank Dieter Oesterhelt (Martinsried, Germany) and Floris P. Buelens (Göttingen, Germany) for valuable discussions on chain-length control and FAS catalytic mechanisms. We are further grateful to Mislav Oreb (Frankfurt, Germany) for helpful advice, and Peter Kötter (Frankfurt, Germany) for providing the double-knockout strain. Work in the lab of E.B. was supported by the Hessian Ministry of Science and Art via the LOEWE research focus 'Integrative fungal research (IPF)'. This work was also supported by a Lichtenberg Grant of the Volkswagen Foundation to M.G. (grant number 85701).

\section{Author contributions}

J.G. and R.P. performed biological studies; J.G., R.P., E.B. and M.G. designed research and analysed data; M.F. performed initial cloning and transformations; J.G., R.P., E.B. and M.G. wrote the manuscript.

\section{Additional information}

Supplementary Information accompanies this paper at http://www.nature.com/ naturecommunications

Competing financial interests: J.G., R.P., E.B. and M.G. are inventors of EP patent application 15162192.7 filed on 1 April 2015, and J.G., E.B. and M.G. are inventors of EP patent application 15174342.4 filed on 26 June 2015, by Goethe-University Frankfurt.

Reprints and permission information is available online at http://npg.nature.com/ reprintsandpermissions/

How to cite this article: Gajewski, J. et al. Engineering fungal de novo fatty acid synthesis for short chain fatty acid production. Nat. Commun. 8, 14650 doi: 10.1038/ncomms14650 (2017).

Publisher's note: Springer Nature remains neutral with regard to jurisdictional claims in published maps and institutional affiliations.

This work is licensed under a Creative Commons Attribution 4.0 International License. The images or other third party material in this article are included in the article's Creative Commons license, unless indicated otherwise in the credit line; if the material is not included under the Creative Commons license, users will need to obtain permission from the license holder to reproduce the material. To view a copy of this license, visit http://creativecommons.org/licenses/by/4.0/ 